Eur. J. Clin. Chem. Clin. Biochem.

Vol. 31, 1993, pp. 645-650

(C) 1993 Walter de Gruyter \& Co. Berlin - New York

\title{
Postmenopausal Hormone Replacement Therapy with Tibolone Decreases Serum Lipoprotein(a)
}

\author{
By W. Haenggi ${ }^{1}, W$. Riesen $^{2}$ and $M . H$. Birkhaeuser ${ }^{1}$ \\ 1 Abteilung für gynäkologische Endokrinologie, Universitäts-Frauenklinik Bern, Switzerland \\ 2 Institut für Klinische Chemie und Hämatologie, Kantonsspital St. Gallen, Switzerland
}

(Received March 31/July 21, 1993)

Summary: Lipoprotein(a) is a cholesterol-rich plasma lipoprotein consisting of LDL and apolipoprotein(a). Apolipoprotein(a) shows structural similarity with plasminogen and thus may interfere with thrombogenesis. Lipoprotein(a) has been shown to be a strong independent risk factor for coronary heart disease.

So far no drug or diet is known to have prominent effects on the serum levels of lipoprotein(a).

In the present study we found a highly significant decrease (in the order of $26 \%$ ) of lipoprotein(a) in 28 women treated for 6 months with Tibolone, compared with an age-matched healthy control group.

Tibolone is a synthetic steroid with gestagenic and weak androgenic and oestrogenic properties, which shows no stimulation of the endometrium. Tibolone also produced a decrease in HDL-cholesterol of $23 \%(\mathrm{p}<0.001)$, a decrease in apolipoprotein A-I of $14 \%(\mathrm{p}<0.001)$ and an increase in apolipoprotein B of $17 \%(\mathrm{p}<0.001)$, whereas the control group showed no significant changes in these quantities.

Tibolone in a daily dose of $2.5 \mathrm{mg}$ is at present the only complete postmenopausal hormone replacement therapy that shows a significant inhibiting influence on serum levels of lipoprotein(a). Its effect on lipoprotein(a) might counterbalance, at least to some extent, the theoretical adverse effect on the other lipoprotein risk factors.

\section{Introduction}

Lipoprotein(a) is a cholesterol-rich plasma lipoprotein consisting of one particle of low density lipoprotein and one molecule of apolipoprotein(a) (1). Apolipoprotein(a) shows a close structural similarity to plasminogen and may compete with plasminogen in fibrinolysis (2). Due to an amino acid substitution at the site of action of the tissue plasminogen activator, apolipoprotein(a), however, may not be split into an active plasmin-like fragment (3).

Lipoprotein(a) has been shown to be a genetically determined independent risk factor for coronary heart disease $(1,4)$ and stroke $(5)$ and its metabolism is independent of that of low density lipoprotein.
Plasma concentrations of lipoprotein(a) vary over a wide range between individuals but they are remarkably constant in any given individual (6).

This study was initiated to get more insight into the different effects of sex steroids on lipoprotein(a), since it is known that lipoproteins are strongly influenced by sex hormones. Low density lipoprotein is known to increase in post menopausal women, probably accounting for the increased cardiovascular risk. Post menopausal hormone replacement therapy with oestrogen has been shown to reduce low density lipoprotein and increase high density lipoprotein levels, with a $40-50 \%$ decrease in the risk of cardiovascular death (7). Little is known, however, about the effects 
of hormone replacement therapy on lipoprotein(a). We therefore looked at changes in plasma lipoproteins (low density lipoprotein-cholesterol, total high density lipoprotein-cholesterol and subfractions, apolipoprotein A-I, apolipoprotein A-II, apolipoprotein B) and lipoprotein(a) in a group of 28 post menopausal women treated for 6 months with Tibolone (Livial ${ }^{\circledR}$; Organon International) and in a control group of untreated volunteers. Tibolone is a synthetic steroid, $(7 \alpha, 17 \alpha)$-17-hydroxy-7-methyl-19-nor-pregn-5(10)-en20-yn-3-one, structurally related to the progestogens, norethynodrel and norethisterone (fig. 1). It shows<smiles>C#C[C@]1(O)CCC2C3C(CCC21I)C1=C(CC(=O)CC1)C[C@H]3C</smiles>

Fig. 1. Chemical structure of Tibolone [Org OD 14: $(7 \alpha, 17 \alpha)$ 17-hydroxy-7-methyl-19-nor-pregn-5(10)-en-20-yn-3one].

weak progestogenic, oestrogenic and androgenic actions in animal tests (8). Tibolone has been shown to be effective in the relief of climacteric symptoms $(9-11)$ and in preventing post menopausal bone loss (12). Tibolone is also effective in the treatment of established postmenopausal osteoporosis (13). A further advantage of Tibolone is the absence of endometrial stimulation $(14,15)$. Thus, in non-hysterectomized women no withdrawal bleeding occurs, an otherwise leading cause for discontinuation of hormone replacement therapy.

\section{Patients and Methods}

\section{Patients}

In 28 apparently healthy, early post menopausal women, serum lipids, lipoprotein(a), low density lipoprotein- and high density lipoprotein-cholesterol, and the apolipoproteins A-I, A-II and $B$ were measured before and after 6 months of hormone substitution with Tibolone $2.5 \mathrm{mg}$ daily. The results were compared with a control group of 28 age-matched postmenopausal volunteers not wishing to receive hormonal substitution therapy. All women participating in the study were recruited among patients consulting our endocrinological outpatient consultation for climacteric symptoms. None of the women had taken oestrogen gestagen medication during at least 2 months prior to the first examination, and none took lipid-lowering or any other type of drug. They were advised not to change their diet and smoking habits.

The major characteristics of the study population are given in table 1 . The two groups did not differ statistically. Menopause was ascertained by serum follitropin values above $30 \mathrm{U} / \mathrm{l}$.

All patients gave their informed consent to participation in the study. The study protocol was approved by the ethical committee of the women's hospital of the University of Berne.
Tab. 1. Characteristics of study population. Data are presented as mean \pm SEM. Body-Mass-Index: weight in $\mathrm{kg} /$ (height in $\mathrm{cm})^{2} \times 10000$.

There was no significant difference between the two groups (Student's $\mathrm{t}$ test: $\mathrm{p}>>_{i} .05$ ).

\begin{tabular}{lrr}
\hline & \multicolumn{1}{c}{ Controls } & \multicolumn{1}{c}{ Tibolone } \\
\hline Number of patients & \multicolumn{1}{c}{28} & \multicolumn{1}{c}{28} \\
Age (a) & $51.9 \pm 0.6$ & $52.9 \pm 0.7$ \\
Duration of menopause (a) & $3.1 \pm 0.4$ & $3.5 \pm 0.4$ \\
Weight (kg) & $64.1 \pm 1.7$ & $68.8 \pm 2.1$ \\
Height (cm) & $164.3 \pm 1.1$ & $163.2 \pm 1.1$ \\
Body-Mass-Index & $23.8 \pm 0.6$ & $24.8 \pm 0.6$ \\
Systolic blood pressure & $124.5 \pm 3.2$ & $126.1 \pm 3.5$ \\
$\quad$ (mmHg) & & \\
Diastolic blood pressure & $78.0 \pm 1.8$ & $79.3 \pm 1.8$ \\
$\quad$ (mmHg) & & \\
Hysterectomized & 9 & 11 \\
Bilateral ovarectomized & 2 & 1 \\
Smoker & 5 & 5 \\
Previous hormonal & 7 & 9 \\
$\quad$ replacement therapy & & \\
\hline
\end{tabular}

\section{Methods}

Fasting blood samples were obtained from all subjects before enrolment in the study and after 6 months. All sera were stored at $-20^{\circ} \mathrm{C}$ until analysed. Total cholesterol and triacylglycerols were measured by enzymatic methods (Boehringer Mạnnheim), high density lipoprotein-cholesterol was determined after precipitation with phosphotungstate $\mathrm{Mg}^{2+}$ (Boehringer Mannheim), and low density lipoprotein-cholesterol was measured after precipitation with an amphiphilic polymer (Bio Mérieux, Lyon) in the washed and re-dissolved precipitate. The high density lipoprotein 2 /high density lipoprotein $\left(\mathrm{HDL}_{2} / \mathrm{HDL}_{3}\right)$ ratio was measured by precipitation with polyethylene glycol according to Kostner (16). Apolipoproteins A-I, A-II and B were measured by immunonephelometry on a Behring Nephelometer Analyser (Behringwerke Marburg). The between-run coefficient of variation for apolipoprotein-A-I was $4.6 \%$ at 1.7 $\mathrm{g} / \mathrm{l}$ and the within-run coefficient of variation was $1.8 \%$. The between-run coefficient of variation for apolipoprotein B was $3.3 \%$ at $1.22 \mathrm{~g} / \mathrm{l}$ and the within-run coefficient of variation was $1.9 \%$. Apolipoprotein values were standardised with reference material from Behring-Werke which is proposed as the WHO standard.

Lipoprotein(a) was measured by immunoradiometric assay (Pharmacia Uppsala). This method was shown not to be affected by storage of samples in the frozen state. The betweenrun coefficient of variation for lipoprotein(a) was $9.7 \%$ at 93 $\mathrm{mg} / \mathrm{l}$.

\section{Statistical analysis}

The comparison of initial values, treatment differences within groups after 6 months and comparison of treatment changes between groups were statistically analysed with Student's test. For lipoprotein(a), which is known for its skewed distribution, logarithmic transformation was performed before testing. Testing was performed two-sided. All p-values below 0.05 were considered to be significant.

\section{Results}

Initial lipid and lipoprotein valụes did not differ between the two groups of volunteers (tab. 2). Lipoprotein(a) concentrations showed the typical skewed var- 


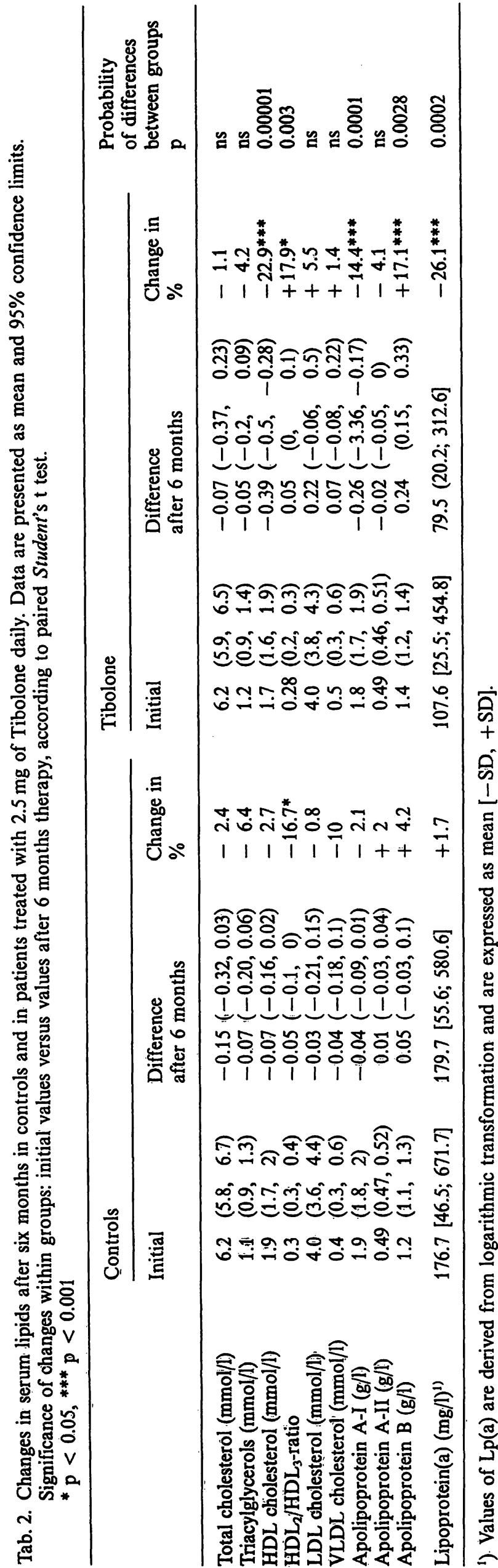

iation among individuals. At the start of the study, 8 of the 28 women in the control group and 4 of the 28 women in the treated group had lipoprotein(a) values above the norm of $300 \mathrm{mg} / \mathrm{l}$, this difference was statistically not significant (Fisher's exact test).

After 6 months of treatment with Tibolone, significant changes were found in the values for high density lipoprotein-cholesterol, the high density lipoprotein ${ }_{2} /$ high density lipoprotein ${ }_{3}$-ratio, apolipoprotein A-I and apolipoprotein B, whereas no significant alterations occurred in total cholesterol, triacylglycerols, low density lipoprotein-cholesterol, very low density lipoprotein-cholesterol and apolipoprotein A-II. High density lipoprotein-cholesterol and apolipoprotein A-I concentrations significantly decreased after Tibolone treatment, whereas apolipoprotein B showed a statistically significant increase (tab. 2). Within the non-substituted group only the high density lipoprotein $2 /$ high density lipoprotein ${ }_{3}$ ratio showed a significant decrease after 6 months. In contrast to apolipoprotein B, lipoprotein(a) showed a decrease of $26.1 \%$ in the treated group, while within the control group an insignificant change of only $1.7 \%$ was observed (tab. 2). When compared between the two groups, the decrease of lipoprotein(a) after 6 months under Tibolone treatment is highly significant $(\mathrm{p}<0.0002)$.

Twenty-four of the 28 lipoprotein(a) values showed a decrease after treatment with Tibolone, the largest decreases occurring in the 7 patients with the highest pre-treatment values.

The mean change of lipoprotein(a) as a percentage of the initial value in the 7 patients with highest initial values (upper quartile) was $0.7 \%$ in the controls and $-42.9 \%$ in the treated group, this difference between the two groups being highly significant $(p<0.004)$.

The individual changes in lipoprotein(a) levels are illustrated in figure 2 .

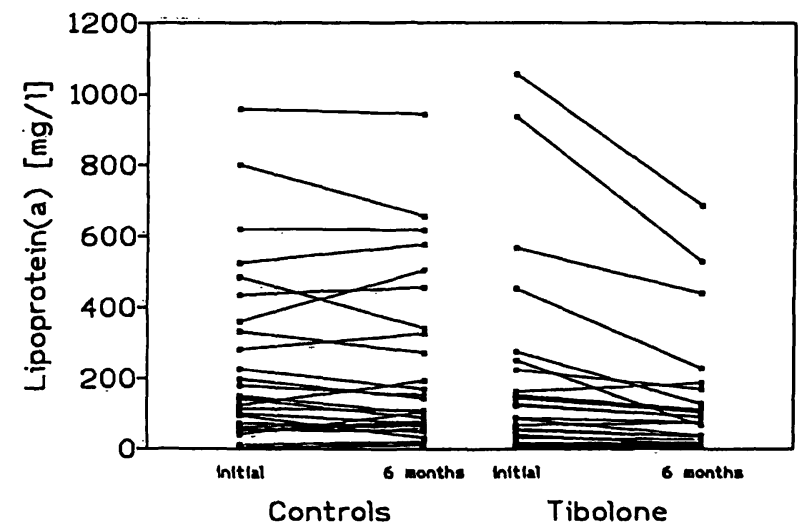

Fig. 2. Individual changes of serum levels of lipoprotein(a) during 6 months in control and treatment groups. 


\section{Discussion}

It is well established that oestrogens and progestins have different effects on lipoproteins. Oestrogens are associated with a decrease in the atherogenic low density lipoprotein fraction and with an increase in the concentration of high density lipoprotein, shown to be negatively associated with coronary heart disease. Tibolone, which was used in the present study, is a synthetic steroid with oestrogenic, progestogenic and weak androgenic activity. We confirmed an already known significant decrease in high density lipoprotein-cholesterol (17) and its major apolipoprotein A-I of $22.9 \%$ and $14.4 \%$, respectively, in the treatment group, probably due to the progestagenic and androgenic properties of Tibolone. Concomitant with the decrease in high density lipoprotein, there was also a significant increase of the high density lipoprotein ${ }_{2} /$ high density lipoprotein $3\left(\mathrm{HDL}_{2} / \mathrm{HDL}_{3}\right)$ ratio in the order of $17.9 \%$, indicating that high density lipoprotein ${ }_{3}$ is decreased to a greater extent than high density lipoprotein ${ }_{2}$. The high density

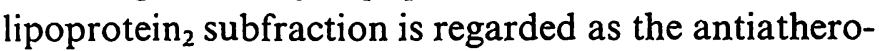
genic fraction of high density lipoprotein-cholesterol (18). Furthermore, Klosterboer et al. demonstrated that after treatment for 3 years, high density lipoprotein-cholesterol returned to pretreatment concentrations (17). However, in view of the important decrease of total high density lipoprotein, an increase of the coronary risk has to be postulated.

On the other hand, recent data from experiments in Cynomolgus monkeys showed that the extent of coronary atherosclerosis was lessened by oestrogen gestagen combination, despite the fact that serum concentrations of high density lipoprotein-cholesterol were lowered (19).

In our treatment group no significant alteration was observed for total cholesterol, triacylclycerols and low density lipoprotein-cholesterol, while apolipoprotein B showed a highly significant increase of $17.1 \%$. Elevated serum concentrations of apolipoprotein B are significantly correlated with a higher risk for cardiovascular disease in women (20). In both groups we found no significant influence on triacylglycerol serum concentrations, but there was a slight decrease of $4 \%$ (Tibolone) and $6 \%$ (controls), compared with the initial values. These results are not in accord with earlier findings $(17,21)$ of a significant decrease $(20 \%)$ of triacylglycerol after Tibolone treatment. Elevated concentrations of triacylglycerols are considered to be associated with a higher risk for cardiovascular disease $(22,23)$.
The main focus of our study, however, was the response of lipoprotein(a), (an independent risk factor for coronary heart disease) to Tibolone treatment. It is difficult to influence the lepvel of lipoprotein(a). Conventional hormone replacement therapy with oral oestrogens only marginally decreased serum concentrations of lipoprotein(a), probably due to a minor influence on its hepatic synthesis (24). A decrease of lipoprotein(a) in the order of $50 \%$, due to combined postmenopausal hormone replacement therapy with $1.25 \mathrm{mg}$ conjugated oestrogens daily plus $10 \mathrm{mg}$ medroxyprogesterone acetate for 10 days a month as described by Soma et al. (25) in 10 patients treated for one year, was not confirmed by the data of Meusing et al. (26). Further indications for an influence of sex steroids on lipoprotein(a) levels are provided by the results of $K u h l$ et al. (27) who found a transitory decrease of lipoprotein(a) in 19 premenopausal women taking an oral contraceptive containing ethinyloestradiol and desogestrel. Lipoprotein(a) decreased during the $3 \mathrm{rd}$ month and reached initial values after 6 months. Two other publications deal with the influence of oestradiol therapy on lipoprotein(a) in men treated for prostatic cancer. Lipoprotein(a) decreased to $50 \%$ after 6 months in 15 men treated with oral ethinyloestradiol $150 \mu \mathrm{g}$ daily (28), whereas the case report of a 67 year old man with familial hypercholesterolaemia treated with $200 \mathrm{mg}$ diethylstilboestrol per day showed a decrease of lipoprotein(a) of more than $80 \%$ after 4 weeks of treatment (29).

Conventional lipid lowering drugs also have little effect on this lipoprotein. The same holds true for diet $(30,31)$. So far, decreases of lipoprotein(a) have been obtained only with niacin and combination of niacin and neomycin $(32,33)$, and by low density lipoprotein apheresis (34).

Statistical analysis of plasma lipoprotein(a) levels is difficult because of their extremely skewed distribution in the white race. In a non-selected population, values above a cut-off-point of $300 \mathrm{mg} / \mathrm{l}$. which are thought to be associated with increased risk for coronary heart disease, are observed in about $20 \%$ of healthy volunteers ( $W$. Riesen: MONICA-Switzerland, unpublished data). In our study group of 56 post menopausal women, lipoprotein(a) concentrations above $300 \mathrm{mg} / \mathrm{l}$ were observed in $21 \%$.

In the group of 28 women treated with Tibolone there was a highly significant decrease in the concentrations of lipoprotein(a). The mean decrease was $26.1 \%$, while in the untreated group a mean increase of $1.7 \%$ 
was observed. Compared with lipid lowering drug treatment, where effects in the order of a $30 \%$ decrease are only seen with very potent lipid regulating agents, the change of lipoprotein(a) with Tibolone treatment is certainly remarkable. These findings are consistent with the results of a pilot study by Farish et al. (35) who demonstrated a significant decrease of the median serum lipoprotein(a) values of $47 \%$ in 9 women treated with $10 \mathrm{mg}$ norethisterone daily for 2 months. Tibolone shows a close structural similarity to norethisterone. These data are compatible with an unexpected constellation of a theoretically unfavourable fall of high density lipoprotein and a favourable change of lipoprotein(a) values.

\section{References}

1. Scanu, A. M. \& Fless, F. M. (1990) Lipoprotein(a). Heterogeneity and biological relevance. J. Clin. Invest. 85, 1709-1715.

2. McLean, J. W., Tomlinson, J. E. \& Kuang, W. J. (1987) cDNA sequence of human apolipoprotein(a) is homologous to plasminogen. Nature $300,132-137$.

3. Loscalzo, J., Weinfeld, M., Fless, G. M. \& Scanu, A. M. (1990) Lipoprotein(a), fibrin binding and plasminogen activation. Arteriosclerosis 10, 240-245.

4. Utermann, G. (1989) The mysteries of lipoprotein(a). Science 246, 904-910.

5. Woo, J., Lau, E., Larm, C. W. K., Kay, R., Teoh, R. \& Wong, H. Y. (1991) Hypertension, lipoprotein(a) and apolipoprotein A-I as risk factors for stroke in the Chinese. Stroke 22, 203-208.

6. Guyton, J. R., Dahlen, G. H., Patsch, W., Kautz, J. A. \& Gotto, A. M. jr. (1985) Relationship of plasma lipoprotein $\mathrm{Lp}$ (a) levels to race and to apolipoprotein B. Arteriosclerosis $5,265-272$.

7. Stampfer, M. J. \& Colditz, G. A. (1991) Estrogen replacement therapy and coronary heart disease: a quantitative assessment of the epidemiologic evidence. Prev. Med. 20, 47-63.

8. De Visser, J., Coert, A., Feenstra, H. \& van der Vies, J. (1984) Endocrinological studies with $(7 \alpha, 17 \alpha)$-17-hydroxy7-methyl-19-norpregn-5(10)-en-20-yn-3-one (Org OD 14). Arzneim. Forschung 34, 1010-1017.

9. Benedek Jasżmann, L. J. (1987) Long-term placebo-controlled efficacy and safety study of Org OD 14 in climacteric women. Maturitas, Suppl. I, 25-33.

10. Kocovic, P. M., Cortes-Prieto, J., Luisi, M., Milojevic, S. \& Franci, F. (1982) Placebo controlled cross-over study of effects of Org OD 14 in menopausal women. Reproduccion $6,81-91$

11. Cittadini, J., Ben, J., Badano, A. R., Denari, H. J., Quiroga, S., Marcus, A. E., Schlaen, I. \& Figueroa-Casas, P. R. (1982) Use of a new steroid (Org OD 14) in the climacteric syndrome. Reproduccion 6, 69-79.

12. Lindsay, R., Hart, D. M. \& Kraszewski, A. (1980) A prospective double-blind trial of synthetic steroid (Org Od 14) for preventing post-menopausal osteoporosis. Br. Med. J. 280, 1207-1209.

13. Geusens, P., Dequeker, J., Gielen, J. \& Schot, L. P. (1991) Non-linear increase in vertebral density induced by a synthetic steroid (Org OD 14) in women with established osteoporosis. Maturitas 13, 155-162.

14. Genazzani, A. R., Benedek Jaszmann, L. J., Hart, D. M., Andolsek, L., Kicovic, P. M. \& Tax, L. (1991) Org OD 14 and the endometrium. Maturitas 13, 243-251.
In conclusion, Tibolone is so far the only complete post menopausal hormone replacement therapy that shows a significant decrease of serum concentration of lipoprotein(a) after an oral intake of $2.5 \mathrm{mg}$ daily. Its effort on lipoprotein(a) concentrations, however, might counterbalance, at least to some extent, the adverse effect of Tibolone on other lipoprotein risk factors such as the important decrease of high density lipoprotein-cholesterol and the significant increase in apolipoprotein B. The clinical long-term effect of Tibolone on the risk of coronary heart disease, known to be elevated in unsubstituted post menopausal women, has to be evaluated in prospective long term studies.

15. Punnonen, R., Luikko, P., Cortes-Prieto, J., Eydam, F., Milojevic, S., Trévoux, R., Chryssikopoulos, E., Franchi, F., Luisi, M. \& Kicovic, P. (1984) Multicentre study of effects of Org OD 14 on endometrium, vaginal cytology and cervical mucus in postmenopausal and oophorectomized women. Maturitas 5, 281-286.

16. Kostner, G. M., Molinari, E. \& Pichler, P. (1985) Evaluation of a new HDL2/HDL3 quantitation based on precipitation with polyethylene glycol. Clin. Chim. Acta 148, 139-147.

17. Kloosterboer, H. J., Benedek Jaszmann, L. J. \& Kicovic, P. M. (1990) Long-term effects of Org OD 14 on lipid metabolism in post-menopausal women. Maturitas 12, $37-42$.

18. Miller, N. E., Hammett, F. \& Saltissi, S. (1981) Relation of angiographically defined coronary artery disease to plasma lipoprotein subfractions and apolipoproteins. $\mathrm{Br}$. Med. J. 282, $1741-1745$.

19. Clarkson, T. B., Shively, C. A., Morgan, T. M., Koritnik, D. R., Adams, M. R. \& Kaplan, J. R. (1990) Oral contraceptives and coronary artery atherosclerosis of cynomolgous monkeys. Obstet. Gynecol. 75, 217-222.

20. Kwiterovich, P. O. Jr., Coresh, J., Smith, H. H., Bachorik, P. S., Derby, C. A. \& Pearson, T. A. (1992) Comparison of the plasma levels of apolipoproteins B and A-1, and other risk factors in men and women with premature coronary artery disease. Am. J. Cardiol. 69, 1015-1021.

21. Farish, E., Fletcher, C. D., Hart, D. M., Lindsay, R. \& Leggate, J. (1984) Org ORD 14: Long-term effects on serum lipoproteins. Maturitas 6, 297-299.

22. Lapidus, L., Bengtsson, C., Lindquist, O., Sigurdsson, J. A. \& Rybo, E. (1985) Triglycerides - main lipid risk factor of cardiovascular disease in women? Acta Med. Scand. 217, $481-489$.

23. Carlson, L. A. \& Böttiger, I. E. (1985) Risk factors of ischaemic heart disease in men and women. Results of the 19-year follow-up of the Stockholm Prospective Study. Acta Med. Scand. 218, 207-211.

24. Lobo, R. A., Notelovitz, M., Bernstein, L., Khan, V. Y.. Ross, R. K. \& Paul, W. L. (1992) Lp(a) lipoprotein: Relationship to cardiovascular disease risk factors, exercise and estrogen. Am. J. Obstet. Gynecol. 166, 1182-1190.

25. Soma, M., Fumagalli, R., Paoletti, R., Meschia, M., Maini, M. C., Crosignani, P., Ghanem, K., Gaubatz, J. \& Morrisett, J. D. (1991) Plasma Lp(a) concentration after oestrogen and progestagen in postmenopausal women (letter). Lancet 337, 612.

26. Meusing, R. A., Miller, V. T., Mills, T. A. \& LaRosa, J. C. (1991) Effects of postmenopausal unopposed estrogen and combined therapy on lipoprotein(a) levels. Arterioscler. Throm. 11,1452a. 
27. Kuhl, H., März, W., Jung-Hoffmann, C., Weber, J., Siekmeier, R. \& Gross, W. (1993) Effect on lipid metabolism of a biphasic desogestrel-containing oral contraceptive: Divergent changes in apolipoprotein $B$ and $E$ and transitory decrease in Lp(a) levels. Contraception 47, 69-83.

28. Henrikkson, P., Angelin, B. \& Berglund, L. (1992) Hormonal regulation of serum $L p(a)$ levels. Opposite effects after estrogen treatment and orchidectomy in males with prostatic carcinoma. J. Clin. Invest. 89, 1166-1171.

29. Hiraga, T., Harada, K., Kobayashi, T. \& Murase, T. (1992) Reduction of serum lipoprotein(a) using estrogen in a man with familial hypercholesterolemia. (Letter). J. Am. Med. Ass. 267, 2328.

30. Brewer, H. B. (1990) Effectiveness of diet and drugs in the treatment of patients with elevated $L p(a)$ levels. In: Lipoprotein(a): 25 Years of Progress (Scanu, A. M.,ed.) New York: Academic Press, pp. 211-220.
31. Kostner, G. M. (1990) The physiological rolc of $L p(a)$. In: Lipoprotein (a): 25 Years of Progress (Scanu, A. M., ed.) New York: Academic Press, pp. 183-204.

32. Gurakar, A., Hoeg, J. M., Kostner, G., Papadopoulos, N. M. \& Brewer, H. B. jr. (1985) Levels of lipoprotein Lp(a) decline with neomycin and niacin treatment. Atherosclerosis $57,293-301$.

33. Carlson, L. A., Hamsten, A. A. \& Asplund, A. (1989) Pronounced lowering of serum levels of lipoprotein $\mathrm{Lp}(\mathrm{a})$ in hyperlipidaemic subjects treated with nicotinic acid. J. Intern. Med. 226, 271-276.

34. Armstrong, V. W., Schleef, J., Thiery, J., Muche, R., SchuffWerner, P., Eisenhauer, T. \& Seidel, D. (1989) Effect of HELP-LDL-apheresis on serum concentrations of human lipoprotein(a): Kinetic analysis of the posttreatment return to baseline levels. Eur. J. Clin. Invest. 19, 235-240.

35. Farish, E., Rolton, H. A., Barnes, J. F. \& Hart, D. M. (1991) Lipoprotein(a) concentrations in postmenopausal women taking norethisterone. Br. Med. J. 303, 694.

Dr. med. Willy Haenggi

Oberarzt

Universitäts-Frauenklinik Bern

Schanzeneckstraße 1

CH-3012 Bern

Switzerland 